\title{
Kinetic Monte Carlo statistics of curvature integration by HACA growth and bay closure reactions for PAH growth in a counterflow diffusion flame
}

\author{
Gustavo Leon ${ }^{\mathrm{a}}$, Angiras Menon ${ }^{\mathrm{a}}$, Laura Pascazio ${ }^{\mathrm{a}}$, Eric Bringley ${ }^{\mathrm{a}}$, Jethro Akroyd ${ }^{\mathrm{a}, \mathrm{b}}$, Markus Kraft ${ }^{\mathrm{a}, \mathrm{b}, \mathrm{c}, *}$ \\ ${ }^{a}$ Department of Chemical Engineering and Biotechnology, University of Cambridge, Philippa Fawcett Drive, Cambridge CB3 OAS, United \\ Kingdom \\ ${ }^{b}$ CARES, Cambridge Centre for Advanced Research and Education in Singapore, 1 Create Way, CREATE Tower, \#05-05, Singapore, 138602 \\ ${ }^{c}$ School of Chemical and Biomedical Engineering, Nanyang Technological University, 62 Nanyang Drive, Singapore, 637459
}

\begin{abstract}
This paper uses a Kinetic Monte Carlo model that includes processes to integrate curvature due to the formation of five- and seven-member rings to simulate polycyclic aromatic hydrocarbons (PAHs) growing in lightly sooting ethylene and acetylene counterflow diffusion flames. The model includes new processes to form seven-member rings via hydrogen-abstraction-acetylene-addition and bay closure reactions on sites containing partially embedded fivemember rings. The model additionally includes bay closure and HACA bay capping reactions for the integration of five-member rings. The mass spectra of PAHs predicted by the model are assessed against experimental data, and the distribution of embedded five-member rings and seven-member rings is studied as a function of spatial location, molecule size and frequency of events sampled in the simulation. The simulations show that the formation of sevenmember rings and the embedding of five-member rings is a competitive process. Both types of rings are observed more frequently as the simulation proceeds from the fuel outlet towards the stagnation plane. Approximately $15 \%$ of the events that integrate curvature resulted in the formation of a seven-member ring coupled to an embedded five-member ring, and the remaining $85 \%$ of events embedded five-member rings via the formation of six-member rings. The proportion of PAHs containing embedded five-member rings and/or seven-member rings is observed to be a function of PAH size, passing through a maximum for PAHs containing 15-20 six-member rings. However, the proportion of PAHs containing both types of ring increases with PAH size, where upwards of $10 \%$ of PAHs containing at least one five-member ring and 15 or more six-member rings also contain a seven-member ring.
\end{abstract}

Keywords: Kinetic Monte Carlo model, curved PAH, counterflow diffusion flame, seven-member ring formation

Colloquium: Soot, nanomaterials and large molecules

Method of Determination: Method 2

Total Word Count: 5500 words, 7.5 pages

Supplemental Material is available for this paper.

\footnotetext{
${ }^{*}$ Corresponding author

Email address: mk306@cam.ac.uk (Markus Kraft)
} 


\section{Introduction}

Curvature induced during the growth of polycyclic aromatic hydrocarbons (PAHs) has important consequences for carbon materials. In the case of soot, it has been shown that curved PAHs possess a dipole moment due to the flexoelectric effect [1] that persists at flame temperatures [2]. Such PAHs have been observed in premixed [3,4] and non-premixed flames [5], and it has been hypothesised that their dipole moments may influence the formation of the first particles [6, 7].

The curvature arises from five- and seven-member rings that are embedded during the growth of a PAH. Five-member rings surrounded by six-member rings results in positive Gauss curvature, corresponding to a bowl-shaped topology. Seven-member rings surrounded by six-member rings results in negative Gauss curvature, corresponding to a saddle-shaped topology.

Five-member rings are found in a variety of carbon materials including nanotubes [8], graphene [9] and fullerenes. Fullerenes have been observed in low pressure benzene flames [10] and have been strongly associated with the presence of curved PAHs [11]. Corannulene (the smallest curved PAH) has been detected in flame-generated soot [11, 12]. High-resolution transmission electron microscopy (HR-TEM) analysis of soot has shown the presence of curved fringes, indicating PAHs containing embedded five-member rings [13, 14]. Partially embedded five-member rings have been directly observed in PAHs using atomic force microscopy, and may be able to lead to fully embedded five-member rings via an acetylene addition step, but observing these embedded five-member rings in soot is challenging because the resulting PAHs are no longer planar [15].

Seven-member rings have been observed in non-graphitising carbon [16], nanotubes [8] and graphene [17]. The Stone-Wales defect, a double pair of five- and seven-member rings, produces local curvature in graphene [18]. Lines of consecutive five- and seven-member rings have been observed in nanoporous carbons [19], and shown to result in different curvatures in different annealed carbons [20]. In graphene, these lines constitute grain boundaries where the orientation of the carbon atoms change [21]. The partially embedded five-member rings observed by Commodo et al. [15] are contained in bays that provides a site for the formation of a seven-member ring next to the five-member ring. HR-TEM analysis of curved fringes also suggests that seven-member rings could be present in soot $[13,14]$.

Kinetic Monte Carlo (KMC) models have been used to simulate PAH growth via the application of a set of transformations and associated rates describing possible growth processes. Frenklach et al. [22] simulated the growth of a graphene edge using the first model that included processes that integrated five-member rings. The model was extended to include five-member ring migration [23], oxygen chemistry that enabled the formation of partially embedded five-member rings [24] and tested under different conditions [25]. Yapp et al. [26] combined a KMC model of the growth of an ensemble of PAHs with a probabilistic model that estimated the Gauss curvature of each PAH as a function of the number of embedded five-member rings.

The above models focused solely on five-member rings as the cause of curvature. The corresponding processes have been studied under a range of conditions. Pope et al. [27] studied the formation of fullerenes via sequential hydrogen-abstraction-acetylene-addition (HACA) and dimerisation reactions. Frenklach and collaborators studied bay capping and competing processes affecting partially embedded five-member rings [28, 29]. Raj [30] investigated the growth of flat and curved PAHs by HACA. However, few studies have considered the formation of seven-member rings. One exception is Kislov et al. [31], who studied a process to create sevenmember rings via two HACA additions on a zig-zag site, but found that it was slow relative to other processes.

Recently, Menon et al. [32] calculated rates for the formation of seven-member rings on bay sites containing five-member rings using density functional theory at the M06-2X/cc-PVTZ//B3LYP/6-311+G(d,p) level of theory. The ring formation mechanisms included hydrogen-abstraction-facilitated, hydrogenaddition-facilitated, carbene formation, and direct cyclisation bay closure processes and closure via HACA growth. The calculated rates showed that the formation of seven-member rings by HACA growth and bay closures proceeded at rates similar to the analogous processes for the formation of five and six-member rings.

The purpose of this paper is to study the development of curvature in PAHs due to HACA growth and bay closure reactions in KMC simulations of a counterflow diffusion flame. The KMC model uses the process rates calculated by Menon et al. [32], for first time enabling simulation of the growth of an ensemble of PAHs that include seven-member rings. The model results are consistent with experimental mass spectra and give insight into the relative abundance and location of PAHs containing embedded five- and seven-member rings. 


\section{Curvature integration processes}

The KMC model used in this work includes two types of process that integrate curvature: The formation of seven-member rings next to existing partially embedded five-member rings and the embedding of five-member rings. These processes are shown in Fig. 1.

Figure 1(a) and (b) show new processes that form seven-member rings via HACA bay capping. The process in Fig. 1(a) results in a seven-member ring coupled to an embedded five-member ring. The process in Fig. 1(b) results in a seven-member ring coupled to a partially embedded five-member ring. The process rates were taken from Menon et al. [32].

Figure 1(c) and (d) show new processes for the closure of seven-member bays adjacent to five-member rings. The process rates were taken from Menon et al. [32].

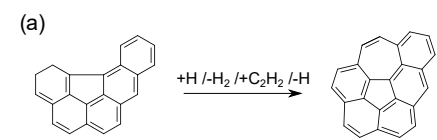

(b)

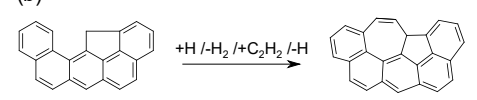

(c)

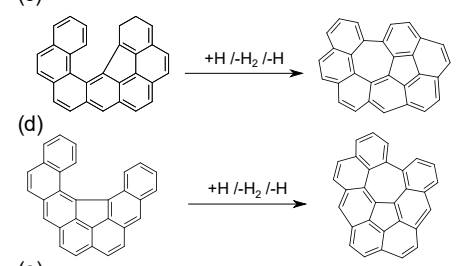

(e)

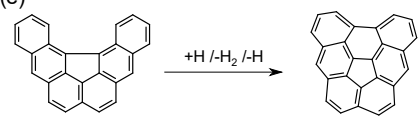

(f)

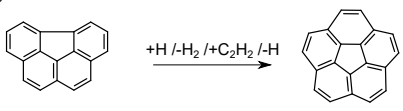

Figure 1: Curvature integration jump processes.

Figure 1(e) shows a new process that embeds a fivemember ring at a six-member bay site that includes a partially embedded five-member ring. There are many possible configurations of such a site. In this work, it was assumed that all configurations proceed at the same rate. The rate is taken by analogy with the most similar process that has been studied in the literature - the closure of a six-member bay site containing only sixmember rings. The rate of this process was first calculated by Raj et al. [33]. In this work we repeated the rate calculation at the B3LYP/6-311+G(d,p) level of theory.
The process was observed to proceed via bay closure following hydrogen abstraction (with a barrier in the range $30-35 \mathrm{kcal} / \mathrm{mol}$ ), or via hydrogen addition, carbene formation or direct cyclisation routes (all with barriers of $\sim 100 \mathrm{kcal} / \mathrm{mol}$ ). The assumption that this rate can be applied to the process in Fig. 1(e) is made on the grounds that hydrogen abstraction is expected to be the most likely route and is expected to have similar rates in both processes, coupled with the observation [32] that the rates of the processes in Fig. 1(a) and (b) are similar and insensitive to the location of the five-member ring, and likewise for the processes in Fig. 1(c) and (d).

Finally, Fig. 1(f) shows a bay capping process that embeds a five-member ring at an armchair site centred on a partially embedded five-member ring. This process is well-known and has been included in previous KMC models. The rate of this process is taken from Raj [30].

Details of the sources for the rates of other processes are provided in the Supplemental Material. Processes that form seven-member rings in the absence of fivemember rings are neglected. This is based on the experimental observation that seven-member rings are typically found next to five-member rings in different carbonaceous materials $[19,21]$.

\section{Computational method}

\subsection{Flame model}

The ethylene and acetylene counter-flow diffusion flames studied by Skeen et al. [34] were selected as targets for this study. These are lightly sooting flames with faint luminosity on the oxidiser side of the stagnation plane. Similar flames have been used for mass spectrometry studies of radical-radical reactions [35] and the spatial dependence of oxygen substituted compounds [36].

A schematic of the ethylene flame is shown in Fig. 2 (and Fig. S1 for the acetylene flame). The flames were simulated using Cantera [37] with the mechanism of Narayanaswamy et al. [38] to solve the one-dimensional continuity, momentum, species and energy equations.

\subsection{Kinetic Monte Carlo model}

A KMC model was used to simulate the growth of PAHs on the fuel side of the flame. The model tracks the spatial coordinates of the carbon atoms and corresponding reactive sites in each PAH. It uses a combination of the steady-state and partial-equilibrium approximations to estimate the rate of reaction at each site. This treatment of the rates has previously been 
shown to give good agreement with deterministic simulations of HACA growth [39]. The temperature and species concentrations from the flame simulations (Section 3.1) were provided as boundary conditions to the KMC model, which simulated the growth of PAHs in a Lagrangian control volume travelling from the fuel inlet (DFFO $=0 \mathrm{~mm}$ ) to just after the sample point (DFFO $=6.17 \mathrm{~mm}$ ). The PAH growth started from pyrene (the largest PAH in the chemcial mechanism), the concentration of which was imposed as a boundary condition from the flame simulations. Methyl additions and oxidation reactions were assumed to be insignificant on the fuel side due to the concentrations of the necessary reactants being low in this domain. Hence, they were neglected in this work. The formation of soot particles by PAH coagulation was not included in the simulation based on the assumption that the PAH growth is dominated by gas-phase reactions, consistent with the selection of lightly sooting flames with faint luminosity.

\section{Results}

\subsection{Mass spectra}

Figure 3 shows simulated mass spectra for the acetylene and ethylene flames versus corresponding experimental data. The peaks heights are scaled to match at $m / z=202$, corresponding to imposing the pyrene concentration as a boundary condition. The maximum number of PAHs in the simulations was 14,060 and 81,500 respectively.

The simulations reproduce the relative abundance of the major peaks reasonably well for the acetylene flame.

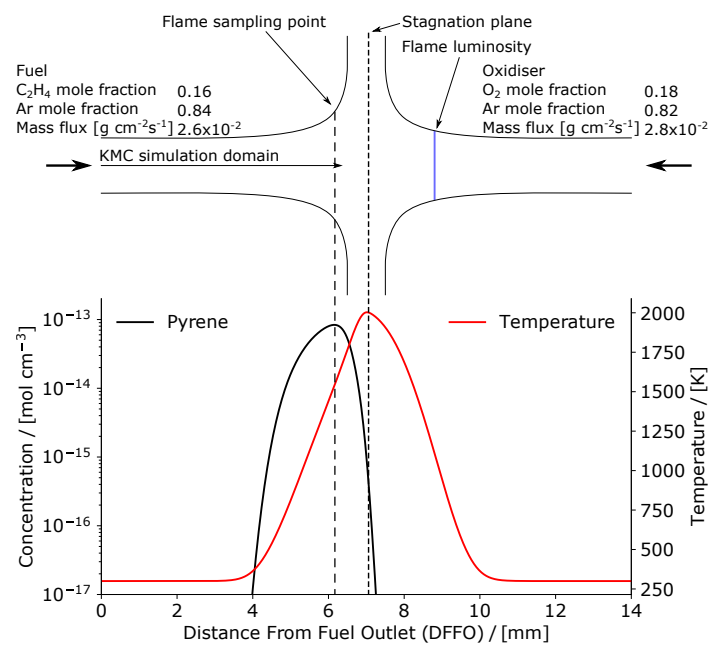

Figure 2: Schematic of the ethylene flame. The concentration of pyrene and temperature show the flame structure.
The level of agreement is less certain for the ethylene flame, where the experimental data are only available up to $m / z=310$. In both cases, and in particular the ethylene flame, the simulations underpredict the peaks for small PAHs, for example at $m / z=226$. This highlights a potential gap in the current modelling approach, where the growth of multiple small PAHs is simulated in the gas-phase chemical mechanism, and then re-simulated rather than imposed in the KMC simulation.

A number of peaks are missing from the simulated spectra. There are several reasons for this. Firstly, some experimentally observed phenomena including methyladdition [40], oxygenated species [36, 41], and isotopes [42] are neglected in the current model. Secondly, the model simulates the growth of PAHs from a single species - pyrene. Given that all the remaining growth processes add two carbons, the simulated spectra currently only include even-carbon-numbered species.

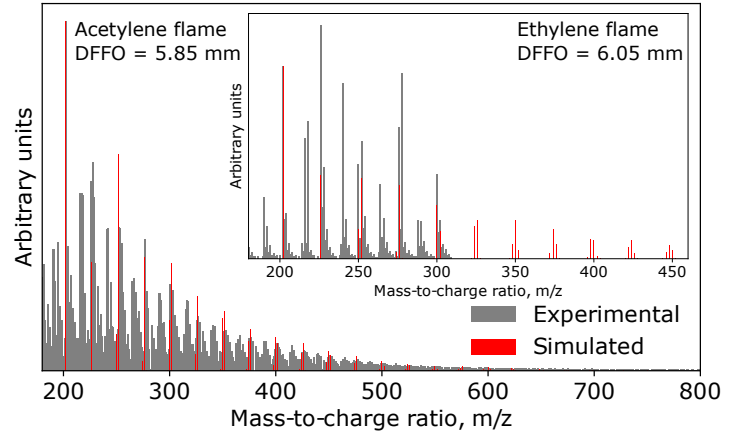

Figure 3: Simulated and experimental [34] mass spectra for the acetylene (main) and ethylene (inset) flames.

\subsection{Integration of five- and seven-member rings}

Figure 4 shows the spatial distribution of PAHs containing five- and seven-member rings in the ethylene flame. The temperature and residence time both contribute to observed distribution and it is not possible to separate each contribution within the current study. The first PAHs with one embedded five-member ring are observed at $\mathrm{DFFO} \approx 5.7 \mathrm{~mm}$. The concentration of these PAHs increases throughout the remainder of the simulation domain. The subsequent addition of five- and seven-member rings occurs via competitive processes that embed a five-member ring either by adding a six- or seven-member ring. Strong correlation is observed between the concentrations of PAHs with two-embedded five member rings and one seven-member ring. Likewise the concentrations of PAHs with four embedded five-member rings and PAHs with two seven-member 
rings. By the end of the simulation domain, a few PAHs containing up to six embedded five-member rings or four seven-member rings can be observed.

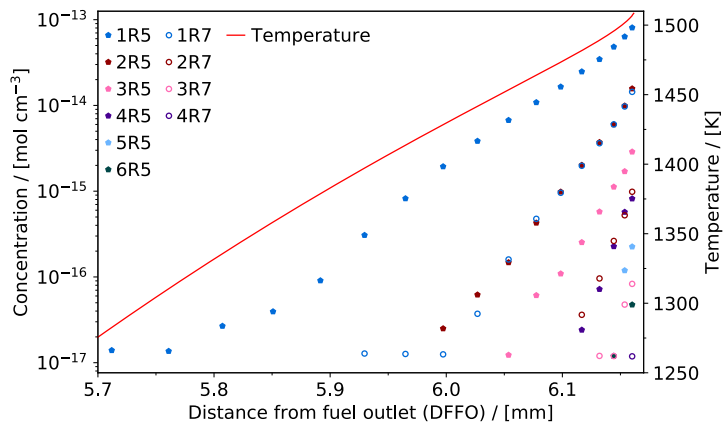

Figure 4: Spatial distribution of temperature and of PAHs containing five- and seven-member rings in the ethylene flame. $n \mathrm{R} 5$ denotes PAHs containing exactly $n$ embedded five-member rings; $n \mathrm{R} 7$ denotes exactly $n$ seven-member rings (embedded or otherwise).

Figure 5 presents a flux diagram showing the relative sampling frequency of the processes that integrate curvature in the ethylene flame. The most frequent processes are HACA (1(f), 47.4\%) and bay closure $(1(\mathrm{e}), 37.3 \%)$ processes that embed five-member rings by adding six-member rings. These occur with similar frequencies. The rate of the bay closure processes is surprisingly high, but can be explained by the nature of the partially embedded five-member ring. Unlike partially embedded five-member rings in armchair sites, which may migrate and desorb [23], bay sites containing partially embedded five-member rings do not allow such migration. Once a bay site containing partially embedded five-member ring appears, it is likely to close and embed the five-member ring.

Both bay closure (1(c) and 1(d), 8.4\%) and HACA (1(a), $1.0 \%$ ) processes simultaneously embed fivemember rings by adding seven-member rings. In this case, the rate of the bay closure is considerably higher than the HACA processes. This is attributed to the inability of a partially embedded five-member ring to migrate from a bay, as above. The remainder of the sevenmember ring additions occur via HACA (1(b), 3.6\%), two thirds of the time followed by the embedding of a five-member ring (1(f), 2.3\%).

Sensitivity analysis suggests that the number of embedded five-member and number of seven-member rings are sensitive to the rates of the processes shown in Figure 5. An increase in the rate of the processes that embed five-member rings results in a decrease in the number of seven-member rings because of competition between these processes for similar sites. An increase in the rate of the processes that form seven-member rings results in a slight increase in the number of embedded five-member rings as well as an increase in the number of seven-member rings because many of these processes simultaneously embed a five-member ring. Details of the sensitivity analysis are provided in the Supplemental Material.

\subsection{Assessing PAH curvature}

The probabilistic model by Yapp et al. [26] estimated the Gauss curvature as a function of the number of embedded five-member rings and six-member rings in a PAH. However, the presence of coupled five- and sevenmember rings that share a common bond result in a molecule that is nearly flat [18], violating the assumptions made in the probabilistic model. The introduction of processes that integrate coupled five- and sevenmember rings in this work allows us to assess the proportion of PAHs for which this occurs.

To assess this, Fig. 6 shows the distributions of the number of PAHs containing different numbers rings at the end of the simulation domain in the ethylene flame. Most of the small PAHs are completely flat. This is expected because a minimum number of five six-member rings needed to embed a five-member ring. The maximum proportion of PAHs with one embedded five-member ring occurs in PAHs with around 15 sixmember rings. This maximum is accompanied by a significant growth in the proportion of PAHs with a second embedded five-member ring. This delayed increase in the number of PAHs that contain a second embedded five-member ring is due to the isolated pentagon rule [43]: adjacent five-member rings are not allowed. This reduces the degrees of freedom when trying to embed a second five-member ring. The maximum proportion of PAHs with seven-member rings occurs in PAHs with $\sim 20$ six-member rings. The reduction in the proportion of PAHs containing ether five- or seven-member rings in large PAHs follows the overall trend in the total number of PAHs.

Overlaid on Fig. 6(a) is a scatter plot of the proportion of PAHs with at least one embedded five-member ring that also contain a seven-member ring, $\phi_{7 \mid 5}$. These PAHs violate the assumptions in the probabilistic model by Yapp et al. [26]. The data become noisy as the number of five-member rings in large species (containing more than 35 six-member rings) decreases. It is observed that the larger a PAH containing an embedded five-member ring, the more likely it is to include a seven-member ring.

The same trend is observed in the proportion of PAHs with at least one seven-member ring that also contain 


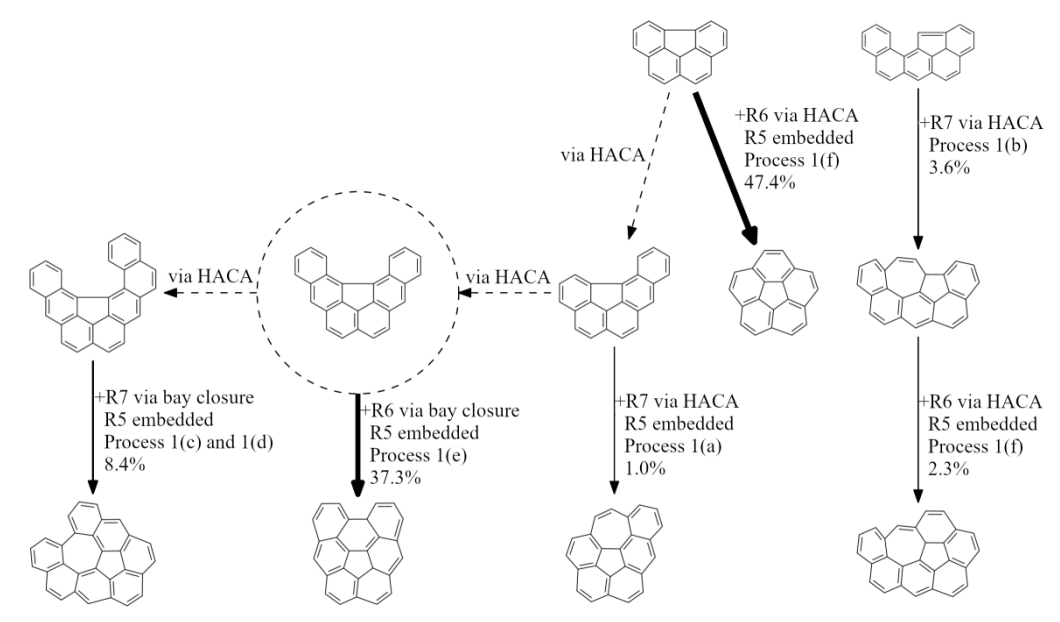

Figure 5: Flux diagram showing the sampling frequency of processes that integrate curvature in the ethylene flame. The solid arrows show processes that integrate curvature. The percentages show the relative sampling frequency of each process. The dashed arrows show processes that add carbon.

at least one embedded five-member ring, $\phi_{5 \mid 7}$ shown in Fig. 6(b). The larger a PAH containing a sevenmember ring, the more likely it is to include a embedded five-member ring. It is also observed that a proportion of PAHs that contain seven-member rings contain no embedded five-member rings. These result from the HACA growth of seven-member rings on partially embedded five-member rings (Fig. 5, Process 1(b), 3.6\%). These five-member rings are eventually embedded as PAHs grows, until all PAHs that contain seven-member rings also contain embedded five-member rings in PAHs with more than 30 six-member rings.

\section{Conclusions}

A KMC model that, for the first time, includes processes to integrate curvature due to the formation of coupled five- and seven-member rings has been used to simulate PAHs growing in ethylene and acetylene counterflow diffusion flames. The simulation results reproduce the major peaks and relative abundances of experimental mass spectra. Including more processes and an more intimate coupling with the gas-phase would allow the simulation of peaks that are currently missing from the simulated spectra.

The addition of five- and seven-member rings occurs via competitiveHACA and bay closure processes. It was observed that approximately $85 \%$ of the events that integrate curvature correspond to the embedding of fivemember rings via the formation of six-member rings, with HACA and bay closures occurring in similar proportions. The remaining $15 \%$ correspond to the formation of seven-member rings coupled to five-member rings, with bay closures occurring approximately twice as often as HACA.

The proportion of PAHs at the end of the simulation domain containing embedded five-member rings and/or seven-member rings is observed to pass through a maximum for PAHs containing 20 six-member rings. The proportion that contains both five- and seven-member rings increases with PAH size. The assumption that the PAHs contain only five and six-member rings made in the probabilistic model introduced by Yapp et al. [26] is increasingly violated as the PAHs increase in size.

The development of a KMC model that includes processes to describe the formation of five- and sevenmember rings by HACA and bay closure processes provides a starting point for future work to model the crosslinking of PAHs. Cross-linking has been suggested to be important for soot formation [44], including specific suggestions about the role of aryl-crosslinks [45], rimbased five-member rings [46], resonantly stabilised radicals [47] and localised $\pi$-radicals [48]. Such crosslinking is expected to create bay sites that require the growth processes implemented in this work.

\section{Acknowledgements}

This work was partly funded by the National Research Foundation (NRF), Prime Minister's Office, Singapore under its CREATE programme. GL is funded by a CONACYT Cambridge Scholarship and wishes to acknowledge the National Council of Science and Technology and the Cambridge Commonwealth Trust. AM gratefully acknowledges Johnson Matthey for financial support. EB was funded by a Gates Cam- 

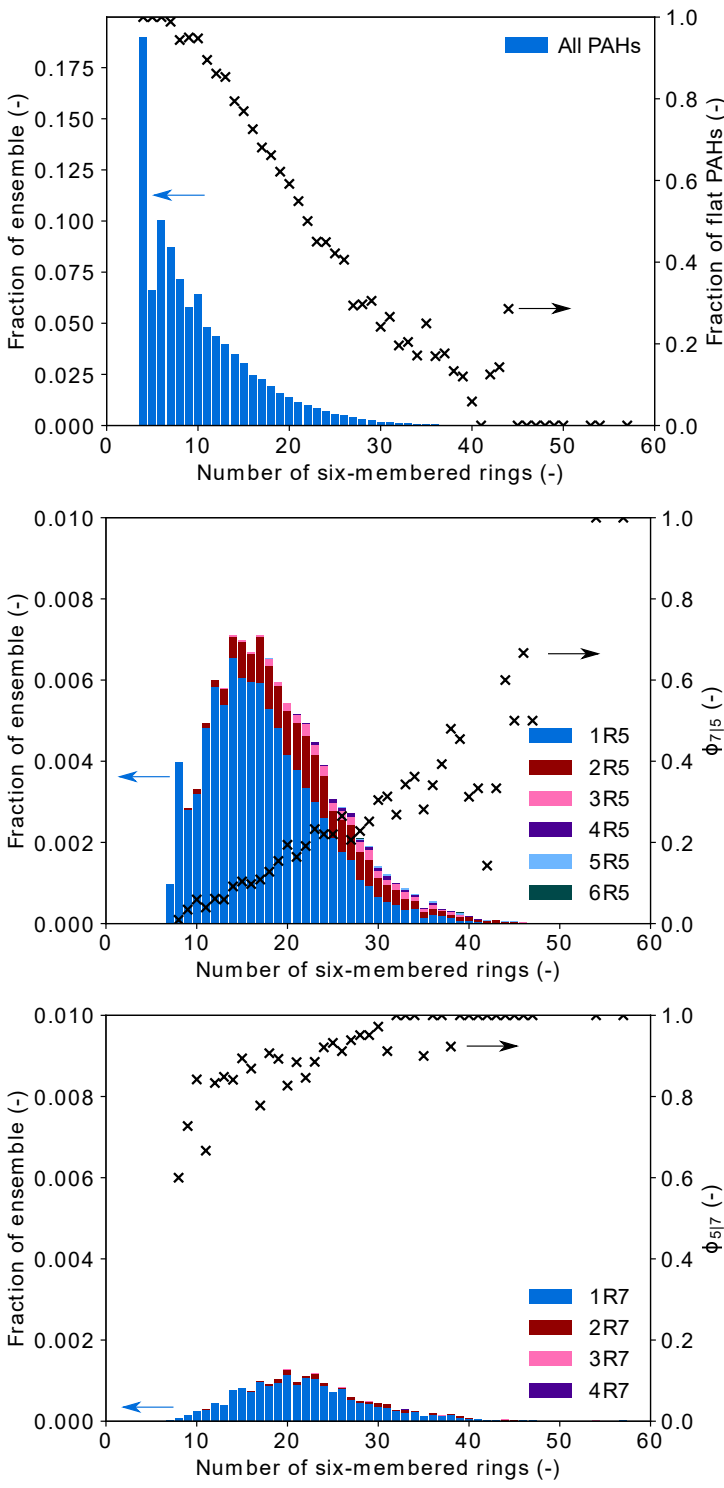

Figure 6: Histograms showing the distributions of the number of fiveand seven-member rings as a function of the number of six-member rings in PAHs in the ethylene flame. The proportion of PAHs with a $j$-member ring that also contain an $i$-member ring is denoted $\phi_{i \mid j}$. $n$ R5 denotes PAHs containing exactly $n$ embedded five-member rings; $n \mathrm{R} 7$ denotes exactly $n$ seven-member rings (embedded or otherwise). $\mathrm{DFFO}=6.17 \mathrm{~mm}$.

bridge Scholarship (OPP1144). MK gratefully acknowledges the support of the Alexander von Humboldt foundation. The authors are grateful to EPSRC (grant number: EP/R029369/1) and ARCHER for financial and computational support as a part of their funding to the UK Consortium on Turbulent Reacting Flows (www.ukctrf.com).

\section{References}

[1] J. W. Martin, R. I. Slavchov, E. K. Y. Yapp, J. Akroyd, S. Mosbach, M. Kraft, The polarization of polycyclic aromatic hydrocarbons curved by pentagon incorporation: The role of the flexoelectric dipole, J. Phys. Chem. C 121 (2017) 27154-27163.

[2] J. W. Martin, A. Menon, C. T. Lao, J. Akroyd, M. Kraft, Dynamic polarity of curved aromatic soot precursors, Combust. Flame 206 (2019) 150-157.

[3] B. Apicella, P. Pré, M. Alfè, A. Ciajolo, V. Gargiulo, C. Russo, A. Tregrossi, D. Deldique, J. Rouzaud, Soot nanostructure evolution in premixed flames by high resolution electron transmission microscopy (HRTEM), Proc. Combust. Inst. 35 (2015) 1895-1902.

[4] W. J. Grieco, J. B. Howard, L. C. Rainey, J. B. V. Sande, Fullerenic carbon in combustion-generated soot, Carbon 38 (2000) 597-614.

[5] J. W. Martin, M. Botero, R. I. Slavchov, K. Bowal, J. Akroyd, S. Mosbach, M. Kraft, Flexoelectricity and the formation of carbon nanoparticles in flames, J. Phys. Chem. C 122 (2018) 22210-22215.

[6] J. W. Martin, K. Bowal, A. Menon, R. I. Slavchov, J. Akroyd, S. Mosbach, M. Kraft, Polar curved polycyclic aromatic hydrocarbons in soot formation, Proc. Combust. Inst. 37 (2019) 1117-1123.

[7] K. Bowal, J. W. Martin, A. J. Misquitta, M. Kraft, Ion-induced soot nucleation using a new potential for curved aromatics, Combust. Sci. Technol. 191 (2019) 747-765.

[8] S. lijima, T. Ichihashi, Y. Ando, Pentagons, heptagons and negative curvature in graphite microtubule growth, Nature 356 (1997) 776-778.

[9] A. W. Robertson, J. H. Warner, Atomic resolution imaging of graphene by transmission electron microscopy, Nanoscale 5 (2013) 4079-4093.

[10] P. Gerhardt, S. Löffler, K. Homann, Polyhedral carbon ions in hydrocarbon flames, Chem. Phys. Lett. 137 (1987) 306-310.

[11] A. L. Lafleur, J. B. Howard, K. Taghizadeh, E. F. Plummer, L. T. Scott, A. Necula, K. C. Swallow, Identification of C20H10 dicyclopentapyrenes in flames: Correlation with corannulene and fullerene formation, J. Phys. Chem. 100 (1996) 17421-17428.

[12] X.-Z. Wu, Y.-R. Yao, M.-M. Chen, H.-R. Tian, J. Xiao, Y.-Y. Xu, M.-S. Lin, L. Abella, C.-B. Tian, C.-L. Gao, Q. Zhang, S.-Y. Xie, R.-B. Huang, L.-S. Zheng, Formation of curvature subunit of carbon in combustion, J. Am. Chem. Soc. 138 (2016) 96299633.

[13] C. Wang, T. Huddle, C.-H. Huang, W. Zhu, R. L. V. Wal, E. H. Lester, J. P. Mathews, Improved quantification of curvature in high-resolution transmission electron microscopy lattice fringe micrographs of soots, Carbon 117 (2017) 174-181.

[14] M. L. Botero, Y. Sheng, J. Akroyd, J. Martin, J. A. Dreyer, W. Yang, M. Kraft, Internal structure of soot particles in a diffusion flame, Carbon 141 (2019) 635-642.

[15] M. Commodo, K. Kaiser, G. D. Falco, P. Minutolo, F. Schulz, A. D'Anna, L. Gross, On the early stages of soot formation: Molecular structure elucidation by high-resolution atomic force microscopy, Combust. Flame 205 (2019) 154-164.

[16] P. J. F. Harris, Fullerene-like models for microporous carbon, J. Mater. Sci. 48 (2013) 565-577.

[17] S. H. Pun, Q. Miao, Toward negatively curved carbons, Acc. Chem. Res. 51 (2018) 1630-1642.

[18] A. I. Podlivaev, L. A. Openov, Dynamics of the Stone-Wales defect in graphene, Phys. Solid State 57 (2015) 820-824.

[19] J. Guo, J. R. Morris, Y. Ihm, C. I. Contescu, N. C. Gallego, G. Duscher, S. J. Pennycook, M. F. Chisholm, Topological 
defects: Origin of nanopores and enhanced adsorption performance in nanoporous carbon, Small 8 (2012) 3283-3288.

[20] J. W. Martin, C. de Tomas, I. Suarez-Martinez, M. Kraft, N. A. Marks, Topology of disordered 3D graphene networks, Phys. Rev. Lett. 123 (2019) 116105.

[21] P. Y. Huang, C. S. Ruiz-Vargas, A. M. van der Zande, W. S. Whitney, M. P. Levendorf, J. W. Kevek, S. Garg, J. S. Alden, C. J. Hustedt, Y. Zhu, J. Park, P. L. McEuen, D. A. Muller, Grains and grain boundaries in single-layer graphene atomic patchwork quilts, Nature 469 (2011) 389-392.

[22] M. Frenklach, C. A. Schuetz, J. Ping, Migration mechanism of aromatic-edge growth, Proc. Combust. Inst. 30 (2005) 13891396.

[23] R. Whitesides, M. Frenklach, Detailed Kinetic Monte Carlo simulations of graphene-edge growth, J. Phys. Chem. A 114 (2010) 689-703.

[24] R. Singh, M. Frenklach, A mechanistic study of the influence of graphene curvature on the rate of high-temperature oxidation by molecular oxygen, Carbon 101 (2016) 203-212.

[25] R. Whitesides, M. Frenklach, Effect of reaction kinetics on graphene-edge morphology and composition, Z. fur Phys. Chem. 229 (2015) 597-614.

[26] E. K. Yapp, C. G. Wells, J. Akroyd, S. Mosbach, R. Xu, M. Kraft, Modelling PAH curvature in laminar premixed flames using a detailed population balance model, Combust. Flame 176 (2017) 172-180.

[27] C. J. Pope, J. A. Marr, J. B. Howard, Chemistry of fullerenes C60 and C70 formation in flames, J. Phys. Chem. 97 (1993) 11001-11013.

[28] X. You, R. Whitesides, D. Zubarev, W. A. Lester, M. Frenklach, Bay-capping reactions: Kinetics and influence on grapheneedge growth, Proc. Combust. Inst. 33 (2011) 685-692.

[29] M. Frenklach, Z. Liu, R. I. Singh, G. R. Galimova, V. N. Azyazov, A. M. Mebel, Detailed, sterically-resolved modeling of soot oxidation: Role of $\mathrm{O}$ atoms, interplay with particle nanostructure, and emergence of inner particle burning, Combust. Flame 188 (2018) 284-306.

[30] A. Raj, Structural effects on the growth of large polycyclic aromatic hydrocarbons by C2H2, Combust. Flame 204 (2019) 331340.

[31] V. V. Kislov, A. I. Sadovnikov, A. M. Mebel, Formation mechanism of polycyclic aromatic hydrocarbons beyond the second aromatic ring, J. Phys. Chem. A 117 (2013) 4794-4816.

[32] A. Menon, G. Leon, J. Akroyd, M. Kraft, A density functional theory study on the kinetics of seven-member ring formation in polyaromatic hydrocarbons, Combust. Flame 217 (2020) 152174.

[33] A. Raj, M. Celnik, R. Shirley, M. Sander, R. Patterson, R. West, M. Kraft, A statistical approach to develop a detailed soot growth model using PAH characteristics, Combust. Flame 156 (2009) 896-913.

[34] S. A. Skeen, H. A. Michelsen, K. R. Wilson, D. M. Popolan, A. Violi, N. Hansen, Near-threshold photoionization mass spectra of combustion-generated high-molecular-weight soot precursors, J. Aerosol Sci. 58 (2013) 86-102.

[35] K. O. Johansson, T. Dillstrom, P. Elvati, M. F. Campbell, P. E. Schrader, D. M. Popolan-Vaida, N. K. Richards-Henderson, K. R. Wilson, A. Violi, H. A. Michelsen, Radical-radical reactions, pyrene nucleation, and incipient soot formation in combustion, Proc. Combust. Inst. 36 (2017) 799-806.

[36] Q. Wang, P. Elvati, D. Kim, K. O. Johansson, P. E. Schrader, H. A. Michelsen, A. Violi, Spatial dependence of the growth of polycyclic aromatic compounds in an ethylene counterflow flame, Carbon 149 (2019) 328-335.

[37] D. G. Goodwin, R. L. Speth, H. K. Moffat, B. W. Weber, Can- tera: An object-oriented software toolkit for chemical kinetics, thermodynamics, and transport processes, https ://www. cantera.org. doi:10.5281/zenodo.1174508, version 2.4.0.

[38] K. Narayanaswamy, G. Blanquart, H. Pitsch, A consistent chemical mechanism for oxidation of substituted aromatic species, Combust. Flame 157 (2010) 1879-1898.

[39] G. Leon, N. Eaves, J. Akroyd, S. Mosbach, M. Kraft, A new methodology to calculate process rates in a Kinetic Monte Carlo model of PAH growth, Combust. Flame 209 (2019) 133-143.

[40] N. Hansen, M. Schenk, K. Moshammer, K. Kohse-Höinghaus, Investigating repetitive reaction pathways for the formation of polycyclic aromatic hydrocarbons in combustion processes, Combust. Flame 180 (2017) 250-261.

[41] C. Irimiea, A. Faccinetto, X. Mercier, I.-K. Ortega, N. Nuns, E. Therssen, P. Desgroux, C. Focsa, Unveiling trends in soot nucleation and growth: When secondary ion mass spectrometry meets statistical analysis, Carbon 144 (2019) 815-830.

[42] M. Schenk, L. Leon, K. Moshammer, P. Oßwald, T. Zeuch, L. Seidel, F. Mauss, K. Kohse-Höinghaus, Detailed mass spectrometric and modeling study of isomeric butene flames, Combust. Flame 160 (2013) 487-503.

[43] H. W. Kroto, The stability of the fullerenes Cn, with $n=24,28$, 32, 36, 50, 60 and 70, Nature 329 (1987) 529-531.

[44] K.-H. Homann, Fullerenes and soot formation - New pathways to large particles in flames, Angew. Chem. Int. Ed. Engl. 37 (1998) 2435-2451.

[45] J. B. Howard, Carbon addition and oxidation reactions in heterogeneous combustion and soot formation, Proc. Combust. Inst. 23 (1991) 1107-1127.

[46] A. D’Anna, A. Violi, A. D'Alessio, A. F. Sarofim, A reaction pathway for nanoparticle formation in rich premixed flames, Combust. Flame 127 (2001) 1995-2003.

[47] K. Johansson, M. Head-Gordon, P. Schrader, K. Wilson, H. Michelsen, Resonance-stabilized hydrocarbon-radical chain reactions may explain soot inception and growth, Science 361 (2018) 997-1000.

[48] J. W. Martin, D. Hou, A. Menon, J. Akroyd, X. You, M. Kraft, Reactivity of polycyclic aromatic hydrocarbon radicals: Implications for soot formation, J. Phys. Chem. C 123 (2019) 26673. 


\section{Supplemental Materials}

Supplemental Material for G. Leon, A. Menon, L. Pascazio, E. Bringley, J. Akroyd, M. Kraft, "Kinetic Monte Carlo statistics of curvature integration by HACA growth and bay closure reactions for PAH growth in a counter-flow diffusion flame, Proc. Combust. Inst. 38 (2021) includes:

- A figure showing a schematic of the acetylene flame.

- Details of the sources for the process rates in the KMC model.

- A sensitivity analysis of the model response with respect to the rates of the processes that embed five-member and form seven-member rings.

- Animations showing the growth of example PAHs. 